\title{
Thromboelastographic Evaluation in Patients with Severe Sepsis or Septic Shock: A Preliminary Analysis
}

\author{
Sokyung Yoon a, JooYen Lim b, Chi-Min Park a,c,*, Dae-Sang Lee d, Jae Berm Park a, \\ Kyoungjin Choi a , Keesang Yoo a, Eunmi Gil a,c, Kyoung Won Yoon a \\ a Department of Surgery, Samsung Medical Center, Sungkyunkwan University School of Medicine, Seoul, Korea \\ ${ }^{b}$ LaPrin Clinics, Seoul, Korea \\ ${ }^{c}$ Department of Critical Care Medicine, Samsung Medical Center, Sungkyunkwan University School of Medicine, Seoul, Korea \\ ${ }^{d}$ Department of Trauma Surgery, Uijeongbu St. Mary's Hospital, College of Medicine, The Catholic University of Korea, Uijeonbu, Korea
}

\section{Article history:}

Received: May 13, 2018

Revised: May 15, 2019

Accepted: October 28, 2019

\section{${ }^{*}$ Corresponding Author:}

Chi-Min Park

Department of Surgery and Critical Care Medicine, Samsung Medical Center, Sungkyunkwan University School of Medicine, Seoul, Korea

E-mail:dr99.park@samsung.com

\section{ORCID}

Sokyung Yoon

Https://orcid.org/0000-0001-6004-664X Chi-Min Park

Https://orcid.org/0000-0002-8496-3546 Dae-Sang Lee

Https://orcid.org/0000-0002-6448-0125 Jae Berm Park

Https://orcid.org/0000-0001-9117-2278

Kyoungjin Choi

Https://orcid.org/0000-0003-2639-2594

Keesang Yoo

Https://orcid.org/0000-0001-6476-8471

Kyoung Won Yoon

Https://orcid.org/0000-0002-9829-5024

\section{ABSTRACT}

Purpose: Thromboelastography (TEG) was investigated for the diagnosis of coagulopathy compared with traditional coagulation tests, in association with disease severity in patients with severe sepsis or septic shock.

Methods: Retrospective data was collected from a single center between January 25th to March 24th, 2016. There were 18 patients with severe sepsis or septic shock admitted to intensive care units included in this study. Laboratory tests including TEG were performed at admission. Disease severity was measured using the Simplified Acute Physiology Score III, Sequential Organ Failure Assessment score, and the level of lactate.

Results: There were 18 patients (61\% males; median age, 60.5 years) who were diagnosed with severe sepsis, or septic shock requiring a norepinephrine infusion $(n=10,55.6 \%)$. Of these, 4 patients had traditional coagulation tests, and TEG profiles which confirmed hypercoagulability. Eight patients had follow-up tests 48 hours post-admission with a Sequential Organ Failure Assessment score of 6.5 (3-9.5) at admission, decreasing to 4 (2-11) after 48 hours (although not significantly lower), however, the lactate level decreased statistically significantly from 2.965 at admission, to $1.405 \mathrm{mmol} / \mathrm{L}$ after 48 hours $(p<0.05)$. The TEG profiles tended to normalize after 48 hours compared with admission, but there was no statistically significant difference.

Conclusion: Coagulopathy with severe sepsis or septic shock patients can be life-threatening, therefore it is important to diagnose coagulopathy early and precisely. TEG can be a feasible tool to confirm coagulopathy with traditional coagulation tests.

Keywords: disease, septic shock, severe sepsis, thromboelastography

\section{Introduction}

Sepsis is a dynamic condition that may progress from a normal coagulation profile to hypercoagulability, hyperfibrinolysis, and ultimately hypocoagulability with increasing disease severity [1]. Sepsis is associated with concurrent activation of coagulation and fibrinolysis, and ensuing downregulation of the fibrinolytic and anticoagulant systems (the latter mainly due to progressive endothelial disruption and damage) [2].
Vascular dysfunction and consumptive coagulopathy may ultimately progress to disseminated intravascular coagulation (DIC), multiple organ failure, and death.

Traditional coagulation tests such as the activated partial thromboplastin time (APTT), prothrombin time, and international normalized ratio (INR) only report hypocoagulability, and although platelet count, plasma fibrinogen, and D-dimer may indicate existing hypercoagulability and hyperfibrinolysis, the result of these tests is qualitative and not functional. 
Hypercoagulability and hyperfibrinolysis in patients with sepsis may be missed by traditional coagulation tests, therefore, several studies have characterized sepsis coagulopathy by viscoelastic hemostatic whole blood clot formation tests such as thromboelastography (TEG) which are quantitative tests [39].

This study investigated TEG, traditional coagulation tests, and disease severity in patients with severe sepsis or septic shock. The primary objective was to investigate the correlation between TEG profiles and traditional coagulation test results in patients with sepsis during their ICU stays, and the secondary objective was to assess its association with disease severity and clinical course.

\section{Materials and Methods}

\section{Patients}

TEG profiles of all patients with severe sepsis or septic shock admitted to surgical and medical intensive care units (ICU) were measured routinely. Patients admitted to the ICU at Sungkyunkwan University Samsung Medical Center with severe sepsis or septic shock between January 25th and March 24th, 2016 were selected for this retrospective study. Patients younger than 20 years old, patients with ongoing bleeding, or taking anticoagulant medication, patients who had trauma, severe hepatic failure, and severe pancreatitis were excluded from this study, resulting in 18 patients being included in this retrospective study. Initial data from traditional laboratory tests and TEG profiles at admission were collected retrospectively, among these, post 48-hour follow-up data of 8 patients were also collected.

Severe sepsis was defined as sepsis plus sepsis-induced organ dysfunction or tissue hypoperfusion; hypotension, lactate above $2.5 \mathrm{mmol} / \mathrm{L}$, urine output $<0.5 \mathrm{~mL} / \mathrm{kg} /$ hour, acute lung injury with $\mathrm{PaO} 2 / \mathrm{Fio} 2<250$, creatinine $>2.0 \mathrm{mg} /$ $\mathrm{dL}$, bilirubin $>2 \mathrm{mg} / \mathrm{dL}$, platelet count $<100 \times 103 / \mu \mathrm{L}$ or coagulopathy (INR > 1.5) [10]. Septic shock was defined as unresponsiveness to fluid resuscitation and requirement of continuous vasopressor therapy to maintain mean arterial blood pressure above $65 \mathrm{mmHg}$. Disease severity was measured using Simplified Acute Physiology Score (SAPS) III, Sequential Organ Failure Assessment (SOFA) score and the level of lactate. The DIC was defined by reference to the following guidelines: British Committee for Standards in Haematology, the Japanese Society of Thrombosis and Hemostasis, and the Italian Society for Thrombosis and Hemostasis [11-14].

Clinical and demographic data at baseline included the SAPS III and the SOFA score that were calculated over the first 6 hours after admission into the ICU. Complete blood cell counts (leukocytes, platelet count), traditional coagulation tests (fibrinogen, D-dimer, INR, aPTT, antithrombin III activity), and serum lactate levels were measured and documented within 6 hours. Blood samples were drawn from an arterial line after discarding the first $5 \mathrm{~mL}$ of blood. After 48 hours, follow-up SOFA scores and laboratory tests were conducted on patients.

This study was reviewed and approved by the Institutional Review Board at Sungkyunkwan University Samsung Medical Center (no.: 2017-10-016).

\section{TEG}

TEG was performed at time of admission and at the 48hour follow-up unless the patient had died or was discharged from the ICU. Whole blood was drawn into $4.5-\mathrm{mL}$ blood vacutainer tubes containing citrate, and TEG analysis was immediately performed by educated personnel in accordance with the standardized procedure and the manufacturer's recommendations [TEG 5,000 Hemostasis Analyzer System (Haemoscope Corporation, Braintree, Miami, FL, USA)] in each ICU. For standard TEG, whole blood was added to a kaolin vial and loaded into the TEG cup containing $\mathrm{CaCl} 2$ for recalcification [15]. The variables recorded were reaction time ( $R$, reference interval as reported by Haemoscope Corporation 4-8 minutes; reflects the rate of initial fibrin formation, time until the first evidence of clot), speed of clot formation ( $K$, reference interval 0-4 minutes; reflects the clot growth kinetics, time taken for certain level of clot strength), angle ( $\alpha$, reference interval $47^{\circ}-74^{\circ}$; reflects the clot growth kinetics, rate/speed of clot formation or rate of fibrin cross-linking), maximum amplitude (MA, reference interval 51-69 mm; reflects the clot strength, maximum clot strength) and lysis after 30 minutes (LY30, reference interval 0-8\%; proportional reduction in amplitude after MA reflecting fibrinolysis) [16,17]. According to TEG alpha angle and MA results, hypercoagulability was defined as alpha angle $>74^{\circ}$ and $\mathrm{MA}>69 \mathrm{~mm}$, hypocoagulability was alpha angle $<47^{\circ}$ and $\mathrm{MA}<51 \mathrm{~mm}$ and normocoagulability was in normal range [1].

\section{Statistical analysis}

The clinical data on the patients were collected retrospectively from electronic medical records. Patients with 1 or more TEG measurements were analyzed.

A clinically improved condition was defined as SOFA score reduced by 2 points or more. Clinically not improved patients were defined as elevated SOFA score or lowered SOFA score of less than 2 points or ongoing shock condition with need for persistent norepinephrine infusion or mortality. The data was presented as median and interquartile range. The Mann-Whitney U test was applied to compare nonparametric variables. Any case where the $p$ value was less than 0.05 was 
considered statistically significant. IBM SPSS statistics 23 (IBM, Armonk, NY, USA) was used for all statistical procedures.

\section{Results}

\section{Basic demographics}

The study included 18 patients (61\% males; median age, 60.5 years) diagnosed with severe sepsis or septic shock requiring norepinephrine infusion ( $n=10,55.6 \%$ ). Among the 18 enrolled patients, 9 patients (50\%) were admitted to the ICU after operative procedures. The median SAPS III score was 53 (45.366.5), and SOFA score was 6.5 (3-9.5). Median serum lactate levels investigated at admission had increased to $2.965 \mathrm{mmol} / \mathrm{L}$ (2.1235.790). Mild leukocytosis, (lower limits of platelet counts) was confirmed with laboratory data. Traditional coagulation tests indicated coagulopathy with high D-dimer, prolonged INR, increased aPTT and decreased antithrombin III activity. Half of the 18 patients were diagnosed with DIC using traditional coagulation tests with low platelet count, low fibrinogen, elevated D-dimer and/or prolonged INR and aPTT. There was no demographic difference between patients admitted to the ICU due to their medical condition or surgical cause (Table 1).

Table 1. Baseline characteristics of study patients at admission $(n=18)$.

\begin{tabular}{lc}
\hline Variable & Value \\
\hline Age $(\mathrm{y})$ & $60.5(46.3-78.5)$ \\
Male sex & $11(61.11)$ \\
\hline Surgical patients & $9(50.00)$ \\
SAPS III & $53(45.3-66.5)$ \\
\hline SOFA score & $6.5(3-9.5)$ \\
Lactate $(\mathrm{mmol} / \mathrm{L})$ & $2.965(2.123-5.790)$ \\
Norepinephrine infusion & $10(55.56)$ \\
Biochemistry at admission & \\
Leukocytes $\left(10^{3} / \mu \mathrm{L}\right)$ & $9.285(3.335-18.165)$ \\
Platelets $\left(10^{3} / \mu \mathrm{L}\right)$ & $139(70.5-199.5)$ \\
Fibrinogen $(\mathrm{mg} / \mathrm{dL})$ & $336(186.5-474)$ \\
D-dimer $(\mathrm{mg} / \mathrm{L})$ & $3.47(1.48-5.57)$ \\
INR & $1.405(1.345-1.745)$ \\
aPTT $(\mathrm{s})$ & $44.45(40.75-52.10)$ \\
Antithrombin III activity $(\%)$ & $51.5(36.00-72.75)$ \\
DIC with traditional lab & $9(50.00)$ \\
\hline
\end{tabular}

Data are presented as medians (IQR, interquartile range) or $n$ (\%).

SAPS III = simplified acute physiology score; SOFA = sequential organ failure assessment; INR = international normalized ratio; aPTT $=$ activated partial thromboplastin time; DIC $=$ disseminated intravascular coagulation.

\section{TEG profiles and correlation with traditional coagulation tests}

According to TEG profiles, 6 patients showed hypocoagulability with low alpha angle and MA, thus 7 patients were confirmed with hypercoagulability with high alpha angle and MA. Four patients were classified as having normocoagulability, with 1 patient with hyperfibrinolysis (LY30 increased above 8\%). After 48 hours, 10 patients improved clinically with SOFA scores lowered by 2 points or more. Three patients needed persistent norepinephrine infusion as they were in ongoing shock. The remaining 5 patients had not improved with elevated SOFA scores or lowered SOFA score less than 2 points. The length of stay (LOS) in the hospital was 13.5 (9.25-33.5) days; ICU LOS was 3.5 (1.75-8.25) days. One of the 18 patients died due to irreversible septic shock (Table 2).

Among the 18 enrolled patients, 6 patients were diagnosed with hypocoagulability using TEG profiles. They were also diagnosed with DIC using traditional coagulation tests, and 4 of the 6 hypocoagulable state patients had improved clinically. However, the other 2 patients had not improved as they both continued to have ongoing shock. Two of the 4 normocoagulable patients were diagnosed with DIC using traditional tests, whilst $25 \%$ of the normocoagulable patients

Table 2. Outcome of study patients $(n=18)$.

\begin{tabular}{lc}
\hline Variable & Value \\
\hline TEG at admission & $4.6(3.15-6.95)$ \\
Reaction time (min) & $1.75(1.2-3.72)$ \\
Clot kinetics (min) & $58.2(41.9-67.18)$ \\
Angle, $\alpha\left(^{\circ}\right)$ & $65.50(46.90-73.35)$ \\
Maximum a mplitude & $0.1(0-0.475)$ \\
(mm) & \\
Lysis index, LY30 (\%) & $6(33.33)$ \\
Diagnosis with TEG & $4(22.22)$ \\
Hypocoagulability & $7(38.89)$ \\
Normocoagulability & $1(5.56)$ \\
Hypercoagulability & $10(66.67)$ \\
Hyperfibrinolysis & $3(16.67)$ \\
Outcome & $3.5(1.75-8.25)$ \\
Clinically improved & $1(5.56)$ \\
Ongoing shock & \\
LOS in hospital (d) & \\
ICU LOS (d) & \\
\hline Mortality & \\
\hline
\end{tabular}

Data are presented as medians (IQR, interquartile range) or $n$ (\%). TEG = thromboelastography; LOS = length of stay; ICU = intensive care units. 
Table 3. Demographics of 18 patients.

\begin{tabular}{lccc}
\hline TEG $(n)$ & $\begin{array}{c}\text { DIC } \\
n(\%)\end{array}$ & $\begin{array}{c}\text { Improved } \\
n(\%)\end{array}$ & $\begin{array}{c}\text { Not improved } \\
n(\%)\end{array}$ \\
\hline Hypocoagulability (6) & $6(100)$ & $4(66.7)$ & $2(33.3)$ \\
Normocoagulability (4) & $2(50)$ & $1(25)$ & $3(75)$ \\
Hypercoagulability (7) & $1(14.3)$ & $5(71.4)$ & $2(28.6)$ \\
Hyperfibrinolysis (1) & & & $1(100)$ \\
\hline
\end{tabular}

DIC $=$ disseminated intravascular coagulation; TEG $=$ thromboelastography. Clinically improved, SOFA score lowered $\geq 2$ points; not improved, elevated SOFA score or lowered SOFA score less than 2 points or ongoing shock (persistent norepinephrine infusion) or mortality.

had improved. Among the 7 patients with hypercoagulability, a single patient was diagnosed with DIC using traditional tests. The remaining 6 patients had results within the normal range of traditional coagulation tests but were confirmed with hypercoagulability using TEG profiles. Five of the 7 patients with hypercoagulability had clinically improved after 48 hours (Table 3).

\section{TEG and clinical outcomes}

This study compared results at admission and at 48 hours follow-up. Of the total of 18 enrolled patients, 8 patients had follow-up laboratory results after 48 hours; of these patients, 50\% were males with a median age of 63.5 years. All 8 patients were admitted to the ICU via the operating room postoperatively. Five patients (62\%) were in septic shock receiving norepinephrine infusions when they were admitted to the ICU. Fibrinogen was significantly elevated from admission to 48 -h post admission ( $\mathrm{p}<0.015$ ). D-dimer, INR and aPTT levels were elevated while antithrombin III activity was lower than the normal range. TEG profiles showed there were no remarkable results but maximum amplitude (MA) was around the lower limits of the normal range. This indicated that clot strength was weak and platelet-fibrin interactions were low. The LOS at the hospital was 20 (10.5-46.25) days and at ICU was 3.5 (2-8.25) days. Disease severity was typically reduced showing a median SOFA score of 6.5 (3-9.5) at admission, and 4 (2-11) after 48 hours, although this was not statistically significant. However, the median lactate levels significantly decreased from 2.965 to $1.405 \mathrm{mmol} / \mathrm{L}(\mathrm{p}<0.05$ ). TEG profiles tended to normalize after 48 hours compared to TEG profiles at admission, but there was no significant difference (Table 4).

The coagulation in the 8 patients are shown in Table 5. Three patients showed hypocoagulability according to their TEG profiles at admission, then after 48 hours, 2 of the 3 patients had clinically improved with a normalization of TEG profiles. Despite the TEG results, all 3 patients were diagnosed with DIC

Table 4. Comparison of results between admission and after 48 hours $(n=8)$.

\begin{tabular}{|c|c|c|c|}
\hline Variable & Admission & After $48 \mathrm{~h}$ & $p$ \\
\hline \multicolumn{4}{|l|}{ Disease severity } \\
\hline SOFA score & $6.5(3-9.5)$ & $4(2-11)$ & 0.442 \\
\hline Lactate $(\mathrm{mmol} / \mathrm{L})$ & $2.965(2.263-3.543)$ & $1.405(1.013-1.982)$ & $0.003^{*}$ \\
\hline \multicolumn{4}{|l|}{ Biochemistry } \\
\hline Platelets $(103 / \mu \mathrm{L})$ & $151(70.5-204.5)$ & $117.5(59-213.8)$ & 0.878 \\
\hline Fibrinogen $(\mathrm{mg} / \mathrm{dL})$ & $234.5(122-392)$ & $445(345.8-540.8)$ & $0.015^{*}$ \\
\hline D-dimer $(\mathrm{mg} / \mathrm{L})$ & $4.44(1.73-5.84)$ & $3.38(3.09-6.14)$ & $>0.999$ \\
\hline INR & $1.40(1.30-1.89)$ & $1.27(1.22-1.51)$ & 0.161 \\
\hline aPTT (s) & $40.85(38.20-51.65)$ & $43.45(38.58-48.80)$ & \\
\hline Antithrombin III activity (\%) & $42.5(32.0-57.0)$ & $43(39-53.8)$ & 0.721 \\
\hline \multicolumn{4}{|l|}{ TEG at admission } \\
\hline Reaction time, $\mathrm{R}$ (min) & $4.55(3.65-8.43)$ & $6(3.9-8.05)$ & 0.574 \\
\hline Clot kinetics, K (min) & $2.05(1.23-3.48)$ & $1.65(1.2-2.53)$ & 0.798 \\
\hline Angle, $\alpha\left(^{\circ}\right)$ & $50.8(43.1-71.3)$ & $67(46.9-72.2)$ & 0.574 \\
\hline Maximum amplitude, MA (mm) & $51.80(40.08-69.20)$ & $66.40(57.25-71.85)$ & 0.279 \\
\hline Lysis index, LY30 (\%) & $0.1(0-0.275)$ & $0.85(0.025-2.350)$ & 0.195 \\
\hline
\end{tabular}

Data are presented as medians (IQR, interquartile range). Two groups were compared by Mann-Whitney U test.

SOFA = sequential organ failure assessment; INR = international normalized ratio; aPTT = activated partial thromboplastin time; TEG = thromboelastography.

${ }^{*} p<0.050$. 
Table 5. Demographics of 8 patients.

\begin{tabular}{|c|c|c|c|}
\hline TEG at admission (n) & TEG after $48 \mathrm{~h}(n)$ & Improved $(n)$ & DIC after $48 \mathrm{~h}$ with traditional tests $(n)$ \\
\hline \multirow[t]{2}{*}{ Hypocoagulability (3) } & Hypocoagulability (1) & 0 & 3 \\
\hline & Normocoagulability (2) & 2 & \\
\hline Normocoagulability (2) & Normocoagulability (2) & 1 & 1 \\
\hline \multirow[t]{2}{*}{ Hypercoagulability (3) } & Normocoagulability (1) & 1 & 0 \\
\hline & Hypercoagulability (2) & 1 & \\
\hline
\end{tabular}

TEG = thromboelastography; DIC $=$ disseminated intravascular coagulation . Clinically improved, SOFA score lowered $\geq 2$ points.

using traditional coagulation tests. Three other patients were within the normal range using traditional tests but TEG profiles showed they had hypercoagulability and 1 of them had not improved clinically.

\section{Discussion}

This study was a preliminary analysis of TEG evaluations compared with traditional coagulation tests. In this study, patients were diagnosed with a variety of coagulopathies like hypocoagulability, hypercoagulability and hyperfibrinolysis using TEG profiles without traditional coagulation tests. There was no significant difference in clinical outcomes between the coagulopathies at admission and after 48 hours. Potentially, coagulopathy could have been misdiagnosed with traditional coagulation tests alone. TEG profiles could help diagnose specific coagulopathies with or without traditional coagulation test results. There was a lack of study populations and data about the clinical outcomes in the present study. It is hard to prove the correlation between TEG profiles and severity of sepsis, status of coagulopathy, clinical outcomes such as LOS or mortality. However, in the 48 hour follow-up, there was an indication of a change in disease severity with the tendency to normalize TEG profiles and more than half of the patients had clinically improved including hypercoagulability in patients, but without a statistical difference. This study showed that TEG profiles could be a diagnostic tool with traditional coagulation tests for coagulopathy, especially for hypercoagulability and hyperfibrinolysis.

TEG, first described by Hartert in 1948 [9], and rotational thromboelastometry are point-of-care methods measuring the kinetics of clot formation in whole blood samples by measuring the viscoelastic properties of the forming clot. Thromboelastometry is a widely accepted method in cardiac and liver transplantation surgery $[18,19]$.

Observations in animal studies and small patient cohorts have suggested that sepsis-evoked alterations in hemostasis might be detected by thromboelastometry $[6,20,21]$. It is unknown, however, whether thromboelastometry variables may predict the outcome of sepsis.

One established method to detect DIC is a sophisticated scoring system encompassing platelet count, INR, fibrinogen, and a fibrinolysis marker. Using such a complex scoring system, groups with low (15\%) and high mortality (40\%) have been discriminated against [22]. Remarkably, however, the results of other studies demonstrate that the presence or absence of a single pathological thromboelastometry finding is associated with marked differences in survival, and allows discrimination of 2 patient subsets with a $58.7 \%$ and $85.7 \%$ 30-day survival, respectively. Furthermore, comparison of thromboelastometry with INR and platelet count in the present study demonstrated that thromboelastometry gave the best prediction of outcome. The fact that early thromboelastometry can detect patients with a worse prognosis suggests that this method may be used to identify patients in which therapeutic modulation of hemostasis might be promising [9]. Furthermore, thromboelastometry may be used to monitor the efficacy of such pharmacological interventions. Both characteristics are important because thromboelastometric results are readily available at the bedside, and an early goal-directed therapy in sepsis is mandatory in the improvement of a prognosis [23].

It is important to state this study has limitations. Firstly, this study was a retrospective study conducted at a single center. Thus, there is more likely to be an inherent bias in the study. Secondly, the study group was too small to make meaningful subgroups including blood product transfusion, renal replacement therapy, extra corporeal membranous oxygenation, and ventilator care. Thirdly, not all the patients received a diagnosis of severe sepsis or septic shock with coagulopathy. As a result, patients with coagulopathy may have been excluded. Fourthly, there was no data about long term mortality. So, in the present study, it was not possible to analyze the correlation between TEG profiles and mortality. This is the first report to date in South Korea evaluating the clinical significance of TEG profiles despite the small study population, however this study showed that TEG profiles can help diagnose a larger variety of coagulopathies than 
traditional coagulation tests in patients' sepsis.

Coagulopathy in severe sepsis or septic shock patients can be a life-threatening condition and it is important to diagnose coagulopathy early and precisely. Traditional coagulation tests had many limitations in detecting different kinds of coagulopathy such as hypercoagulability and hyperfibrinolysis. TEG can be a feasible tool to confirm coagulopathy alongside traditional coagulation tests [24].

\section{Conclusion}

A variety of coagulopathies such as hypocoagulability, hypercoagulability and hyperfibrinolysis that cannot be detected using traditional tests can be detected using TEG profiles in sepsis patients. In addition, TEG profiles may normalize with recovery from shock and this change in TEG profile and coagulation may not correlate to the change in severity and clinical course over the 48-hour period. A largescale prospective study is needed to investigate whether TEG guided diagnosis of treatments can improve the prognosis of sepsis patients.

\section{Conflicts of Interest}

The authors have no conflicts of interest to declare.

\section{References}

[1] Ostrowski SR, Windeløv NA, Ibsen M, Haase N, Perner A, Johansson PI. Consecutive thrombelastography clot strength profiles in patients with severe sepsis and their association with 28-day mortality: A prospective study. J Crit Care 2013;28(3):317.e1-11.

[2] Schouten M, Wiersinga WJ, Levi M, van der Poll T. Inflammation, endothelium, and coagulation in sepsis. J Leukoc Biol 2008;83(3):536-45.

[3] Collins PW, Macchiavello LI, Lewis SJ, Macartney NJ, Saayman AG, Luddington R, et al. Global tests of haemostasis in critically ill patients with severe sepsis syndrome compared to controls. Br J Haematol 2006;135(2):220-7.

[4] Gonano C, Sitzwohl C, Meitner E, Weinstabl C, Kettner SC. Four-day antithrombin therapy does not seem to attenuate hypercoagulability in patients suffering from sepsis. Crit Care 2006;10(6):R160.

[5] Daudel F, Kessler U, Folly H, Lienert JS, Takala J, Jakob SM. Thromboelastometry for the assessment of coagulation abnormalities in early and established adult sepsis: A prospective cohort study. Crit Care 2009;13(2):R42.
[6] Sivula M, Pettila V, Niemi TT, Varpula M, Kuitunen AH. Thromboelastometry in patients with severe sepsis and disseminated intravascular coagulation. Blood Coagul Fibrinolysis 2009;20(6):419-26.

[7] Johansson PI, Stensballe J, Vindelov N, Perner A, Espersen K. Hypocoagulability, as evaluated by thrombelastography, at admission to the ICU is associated with increased 30-day mortality. Blood Coagul Fibrinolysis 2010;21(2):168-74.

[8] Sharma P, Saxena R. A novel thromboelastographic score to identify overt disseminated intravascular coagulation resulting in a hypocoagulable state. Am J Clin Pathol 2010;134(1):97-102.

[9] Adamzik M, Langemeier T, Frey UH, Gorlinger K, Saner F, Eggebrecht H, et al. Comparison of thrombelastometry with simplified acute physiology score II and sequential organ failure assessment scores for the prediction of 30-day survival: A cohort study. Shock 2011;35(4):339-42.

[10] American College of Chest Physicians/Society of Critical Care Medicine Consensus Conference: definitions for sepsis and organ failure and guidelines for the use of innovative therapies in sepsis. Crit Care Med 1992;20(6):864-74.

[11] Levi M, Toh CH, Thachil J, Watson HG. Guidelines for the diagnosis and management of disseminated intravascular coagulation. British Committee for Standards in Haematology. Br J Haematol 2009;145(1):2433.

[12] Taylor Jr FB, Toh CH, Hoots WK, Wada H, Levi M. Towards definition, clinical and laboratory criteria, and a scoring system for disseminated intravascular coagulation. Thromb Haemost 2001;86(5):1327-30.

[13] Wada H, Asakura H, Okamoto K, Iba T, Uchiyama T, Kawasugi K, et al. Expert consensus for the treatment of disseminated intravascular coagulation in Japan. Thromb Res 2010;125(1):6-11.

[14] Di Nisio M, Baudo F, Cosmi B, D'Angelo A, De Gasperi A, Malato A, et al Diagnosis and treatment of disseminated intravascular coagulation: Guidelines of the Italian Society for Haemostasis and Thrombosis (SISET) Thromb Res 2012;129(5):e177-84.

[15] Thakur M, Ahmed AB. A Review of Thromboelastography. Int J Periop Ultrasound Appl Technol 2012;1(1):25-9.

[16] Department of Surgical Education, Orlando Regional Medical Center [Internet]. Thromboelastography (TEG) in Trauma. 2014 [cited 2017 Jan 15]. Available from: http://www.surgicalcriticalcare.net/Guidelines/ TEG\%202014.pdf.

[17] Abdelfattah K, Cripps MW. Thromboelastography and Rotational Thromboelastometry use in trauma. Int J Surg 2016;33(Pt B):196-201.

[18] Gillies BS. Thromboelastography and liver transplantation. Semin Thromb Hemost 1995;21 Suppl 4:45-9.

[19] Dunning J, Versteegh M, Fabbri A, Pavie A, Kolh P, Lockowandt U, et al. Guideline on antiplatelet and anticoagulation management in cardiac surgery. Eur J Cardiothorac Surg 2008;34(1):73-92.

[20] Zacharowski K, Sucker C, Zacharowski P, Hartmann M. Thrombelastography for the monitoring of lipopolysaccharide induced activation of coagulation. Thromb Haemost 2006;95(3):557-61.

[21] Velik-Salchner C, Streif W, Innerhofer P, Maier S, Knotzer H, Pajk W, et al. Endotoxinemia-induced changes in coagulation as measured by rotation thrombelastometry technique and conventional laboratory tests: Results of a pilot study on pigs. Blood Coagul Fibrinolysis 2009;20(1):41-6.

[22] Bakhtiari K, Meijers JC, de Jonge E, Levi M. Prospective validation of the International Society of Thrombosis and Haemostasis scoring system for disseminated intravascular coagulation. Crit Care Med. 2004;32(12):241621.

[23] Lin SM, Huang CD, Lin HC, Liu CY, Wang CH, Kuo HP. A modified goaldirected protocol improves clinical outcomes in intensive care unit patients with septic shock: A randomized controlled trial. Shock 2006;26(6):551-7.

[24] Haase N, Ostrowski SR, Wetterslev J, Lange T, Moller MH, Tousi H, et al. Thromboelastography in patients with severe sepsis: A prospective cohort study. Intensive Care Med 2015;41(1):77-85. 